\title{
Effects of long-term nonylphenol exposure on myocardial fibrosis and cardiac function in rats
}

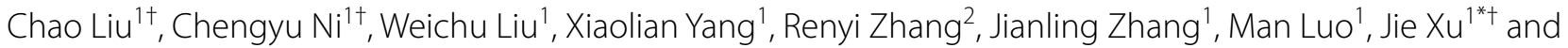
Jie $\mathrm{Yu}^{1 *+}$ (1)

\begin{abstract}
Background: Myocardial fibrosis is a critical pathological basis for the poor prognosis of cardiovascular diseases. Studies have found that myocardial fibrosis is closely associated with exposure to environmental estrogens such as nonylphenol (NP), as a representative of environmental estrogens. The aim of this study was to examine the effects of NP chronic exposure on myocardial fibrosis as well as cardiac structure and function. Forty Sprague-Dawley rats were randomly divided into four groups $(n=10)$ : control group $(C)$, low NP dose $(0.4 \mathrm{mg} / \mathrm{kg}, \mathrm{L})$, medium NP dose (4 mg/kg, $\mathrm{M})$, and high NP dose $(40 \mathrm{mg} / \mathrm{kg}, \mathrm{H})$ groups. The NP dose groups were gavaged with NP for 180 days.
\end{abstract}

Results: The NP level in the heart of the NP groups was significantly higher than those in the control group $(F=43.658, P<0.001)$. Serum aspartate aminotransferase (AST), creatine kinase (CK), creatine kinase isozyme (CK$\mathrm{MB})$, lactate dehydrogenase $(\mathrm{LDH})$ and a-hydroxybutyrate dehydrogenase $(\mathrm{a}-\mathrm{HBDH})$ significantly increased in the NP groups compared with the control group $(P<0.05)$. Histopathological examination of the heart biopsy illustrates that in the medium and high NP groups, the fibrous connective tissue had a disordered and loose gridding shape, muscle fibers had fractured, and muscle fibers were loose with a widened gap. Extensive inflammatory cell infiltration and fibroblast proliferation in the myocardial interstitium were also found. With increasing NP dose, the degree of muscle fiber loosing and disorder became more significant in the NP-treatment groups, and the collagen volume fraction (CVF) was higher than that in the control group $(P<0.01)$. Compared with the control group, the expression of collagen I and collagen III increased significantly in the medium and high NP groups $(P<0.05)$. The values of the systolic thickness of the left ventricular anterior wall (LVAWs), the diastolic thickness of the left ventricular posterior wall (LVPWd), the systolic thickness of the left ventricular posterior wall (LVPWs), and the left ventricular anterior wall (LVAWd) in the NP groups are were slightly lower than those in of the control group. The values of left ventricular end systolic dimensions (LVIDs) in the NP groups increased compared with the control group.

Conclusions: Long-term NP exposure could lead to fibrosis in the rat myocardium, which is characterized by increased expressions of myocardial collagen I and collagen III, as well as elevated cardiac enzymes. In addition, the cardiac structure was affected and changes were observed in the thinner ventricular wall and as an enlarged ventricular cavity.

\footnotetext{
*Correspondence: Xujie360@sina.com;Yujie128@hotmail.com

${ }^{\dagger}$ Chao Liu and Chengyu Ni contributed equally to this paper

${ }^{\dagger} \mathrm{Jie}$ Yu and Jie Xu are co-corresponding authors who contributed equally

to this study

${ }^{1}$ School of Public Health, Zunyi Medical University, Zunyi, Guizhou

563000, People's Republic of China

Full list of author information is available at the end of the article
}

\section{Springer Open}

(c) The Author(s) 2021. Open Access This article is licensed under a Creative Commons Attribution 4.0 International License, which permits use, sharing, adaptation, distribution and reproduction in any medium or format, as long as you give appropriate credit to the original author(s) and the source, provide a link to the Creative Commons licence, and indicate if changes were made. The images or other third party material in this article are included in the article's Creative Commons licence, unless indicated otherwise in a credit line to the material. If material is not included in the article's Creative Commons licence and your intended use is not permitted by statutory regulation or exceeds the permitted use, you will need to obtain permission directly from the copyright holder. To view a copy of this licence, visit http://creativecommons.org/licenses/by/4.0/. 


\section{Highlights}

1. Chronic NP exposure induced myocardial fibrosis and cardiac functional damage.

2. NP increased the expression of myocardial collagen I and III and cardiac enzymes.

3. NP caused cardiac structure changes with thinner ventricular wall and enlarged ventricular cavity.

Keywords: Nonylphenol, Myocardial fibrosis, Collagen I, Collagen III, Cardiac Doppler ultrasound, Masson staining

\section{Background}

Myocardial fibrosis is referred to as an excessive accumulation of stromal cells and extracellular matrix proteins in the myocardium. Progressive fibrosis causes stiffening of the cardiac tissue and affects conduction of electrical impulses, leading to heart failures in a broad range of cardiac conditions [1]. Myocardial fibrosis is a critical pathological basis and an important influencing factor for the poor prognosis of cardiovascular diseases, such as chronic heart failure [2-4]. Studies have found that myocardial fibrosis is closely associated with exposure to environmental endocrine disruptors (EEDs). For example, bisphenol A (BPA) up-regulated collagen I and collagen III expressions in cardiac fibroblasts of male rats by activating the ERK1/2 pathway, which resulted in myocardial fibrosis, similar to the mechanism of $17 \beta$-estradiol increasing collagen in cardiac fibroblasts $[5,6]$. The dialkylphosphate metabolites of organophosphorus pesticides could exacerbate parasitic infection in acute phase and induce myocardial fibrosis [7]. The deltamethrin insecticide induced myocardial fibrosis in rats via oxidative stress-related inflammation and apoptosis by the NF-kB signaling pathway [8]. Subacute endosulfan poisoning could cause cell necrosis, inflammatory response, and myocardial fibrosis [9]. Recently, the environmental pollution of EEDs had attracted increasing attention $[10,11]$. EEDs include more than 70 substances, such as nonylphenol (NP), BPA, hexachlorobiphenyl, and other chemicals with similar estrogenic activity in organisms. It has been proved that EEDs have estrogenic activity similar to the in vivo estrogens, which simulate physiological and biochemical effects of endogenous estrogen and interfere with the normal endocrine system after entering the body [12].

As a representative of EEDs, nonylphenol (NP) is an important chemical material and intermediate. NP is mainly produced by environmental organic pollutants in the fields of surfactants, textile dyeing auxiliaries, lubricant additive, and pesticide emulsifiers, with high lipid solubility and bioaccumulation [13]. Exposure to the emerging contaminant NP is ubiquitous. Our prior study demonstrated that the pollution of NP in Xiangjiang River, tap water, and aquatic in Zunyi city of Guizhou province in China belongs to moderate or severe level in the world [14]. NP can be detected in human blood, urine, milk, fat, and semen $[15,16]$. Many current animal studies have demonstrated that NP exerted a direct toxic effect on the heart, affecting cardiac contractility and L-type $\mathrm{Ca}^{2+}$ channel currents in a non-monotonic manner mainly through the G-protein-coupled receptor 30 [17]. However, whether long-term environmental exposure to such environmental estrogens causes changes in cardiac function and structure urgently needs to be investigated.

Cardiac Doppler ultrasound is widely used for cardiac evaluation and examination in the clinic, and is an important tool for the detection of the cardiac structure and function [18]. Whether the structure and function of the left ventricle of the heart is affected can be assessed with the help of Doppler ultrasound. For myocardial zymography, lactate dehydrogenase (LDH), aspartate aminotransferase (AST), alanine transaminase (ALT), serum creatine kinase isozyme (CK-MB), and hydroxybutyrate dehydrogenase (HDBH) are the most studied myocardial zymography indicators in the clinic. The increased level of a single indicator has no strong directivity toward myocardial injury, but increased levels of multiple indicators can be used to accurately determine the severity of the injury and the effectiveness of clinical treatment [19-21]. Myocardial enzyme and protein levels in peripheral blood can reflect the degree of myocardial ischemic necrosis, and may provide clues for the search for NP-induced MF.

To determine whether the heart is a likely target organ for NP to exert its toxic effects, and whether exposure to NP at environmental concentration would damage cardiac function and cause myocardial fibrosis, therefore, this study detected for the first time the concentration of accumulated NP in the heart, and 
investigated the structural changes in the heart by cardiac Doppler ultrasound. Ultrastructural changes of myocardial fibrosis were qualitatively and quantitatively observed by HE, Sirius red, and Masson staining. Moreover, changes in serum cardiac enzymes were measured to determine the severity of myocardial injury. This study clarified the effects of NP chronic exposure on myocardial fibrosis and cardiac structure and function, and provided a scientific basis of the drug target for the treatment of myocardial fibrosis induced by environmental NP exposure.

\section{Materials and methods Reagents}

The nonylphenol (99\% purity, product code: BT5655) was purchased from the Shandong Xiya Chemical Industry co. LTD (Shandong, China). Masson staining kit (Code: G1005), Sirius red staining kit (Code: G1006), and hematoxylin and eosin staining kit (Code: G1018) were purchased from the Wuhan Guge biological co., LTD (Hubei, China). BCA protein Quantitation Kit was purchased from the Beijing Solarbio Science and Technology Co., Ltd. (Beijing, China). $\beta$-Tubulin antibody, anti-collagen type I antibody and anti-collagen type III antibody were purchased from Abcam (Cambridge, MA, USA). HP-1100 high-performance liquid chromatography (HPLC) was purchased from the Agilent Technologies (Palo Alto, CA, USA). AU5800 automatic biochemical analyzer was purchased from the Beckman Coulter (Indianapolis, IN, USA). Vevo 2100 high-resolution ultrasound imaging system was purchased from the VisualSonics (VisualSonics, Inc, USA). Eclipse optical microscope was purchased from the Nikon (Nikon, Japan). All other chemicals were commercially available. All chemical purities were at least $99 \%$.

\section{Grouping and poisoning}

After 1 week of acclimation, 40 Sprague-Dawley (SD) rats, aged 4 weeks $(210 \pm 10 \mathrm{~g})$, were obtained from Animal Center of the Tianqin Biotechnology Co., Ltd. (License No: SCXK2014-0011, Changsha, China). The rats were randomly divided into four groups $(n=10)$ : control group (C), low NP dose (L), medium NP dose $(\mathrm{M})$, and high NP dose $(\mathrm{H})$ groups. Every morning from 8:00 to 9:00, the control group received $5 \mathrm{ml} / \mathrm{kg}$ corn oil by gavage under $12 \mathrm{~h}$ fasting. The low NP, medium NP, and high NP dose groups were gavaged with NP of $0.4 \mathrm{mg} / \mathrm{kg}, 4 \mathrm{mg} / \mathrm{kg}$, and $40 \mathrm{mg} / \mathrm{kg}$, respectively, for 180 days.

Feeding condition: all rats were raised in conventional plastic cages with free access to water at temperature of $24 \pm 2{ }^{\circ} \mathrm{C}$, humidity of $50 \pm 10 \%$, and light cycle of $12 \mathrm{~h}$

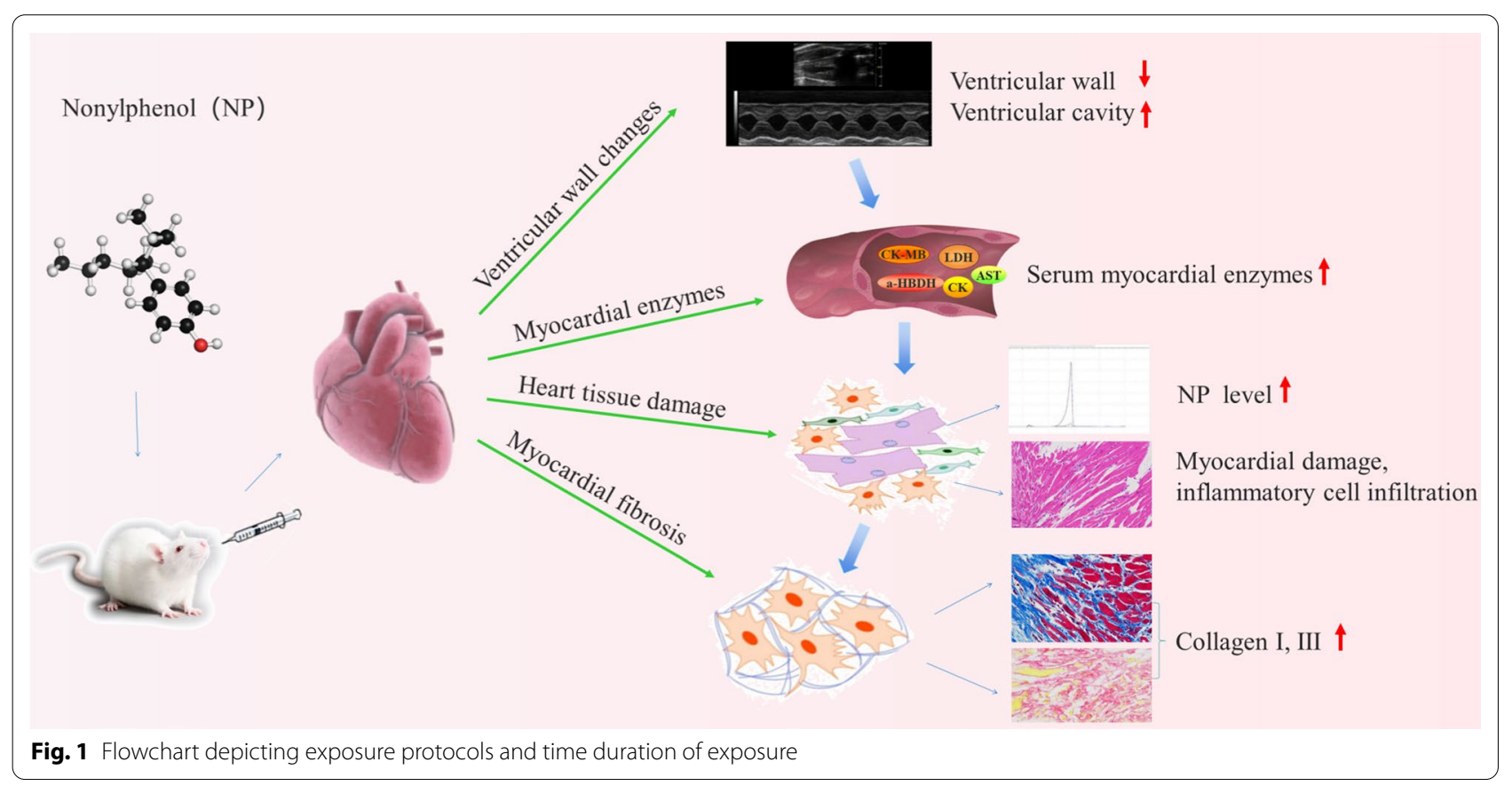


light $/ 12 \mathrm{~h}$ dark. The dose was designed based on the tolerable daily intake of NP of $5 \mathrm{~g} / \mathrm{kg}$.bw/day as proposed by the Danish Institute for Toxicological Safety and Toxicology. The safety coefficient of humans and rats is 1:100; therefore, the tolerated dose of NP in rats was $0.5 \mathrm{~g} /$ $\mathrm{kg} . \mathrm{bw} /$ day. The lowest dose in this study was slightly below the lower safe dose limit, and the medium and high doses were set to 10 times and 100 times of the lowest dose. All methods were performed in accordance with guidelines and regulations of the Zunyi Medical University (No: 2015-2-011). A flowchart depicting exposure protocols for the rats and time duration of exposure is shown in Fig. 1.

\section{NP level detection by HPLC}

The dispensed heart tissue (left ventricle) was taken out from the refrigerator at $-80{ }^{\circ} \mathrm{C}$, partially thawed on the ice, with the excess water absorbed on the filter paper. A total of $10 \mathrm{mg}$ heart tissue was chopped, homogenized with hexane/diethyl ether, and centrifuged (3000r/ min). The supernatant was evaporated in a water bath. The sample was assessed by high-performance liquid chromatography (HPLC). The peak time of standard sample of NP is determined as $3 \mathrm{~min}$ and $24 \mathrm{~s}$. Chromatographic conditions: column (ECLIPSE XD8-C18, $150 \mathrm{~mm} \times 460 \mathrm{~mm}, 5 \mathrm{~mm}$, Agilent, USA). The mobile phase A consisted of acetonitrile and mobile phase $B$ consisted of $0.1 \%$ glacial acetic acid $(\mathrm{A} / \mathrm{B}=85 / 15, \mathrm{v} / \mathrm{v})$ at an injection volume of $10 \mu \mathrm{L}$. The excitation and emission wavelengths were $275 \mathrm{~nm}$ and $312 \mathrm{~nm}$, respectively.

\section{Sample processing and staining}

At the end of NP poisoning, after rats had been anesthetized with $20 \%$ urethane $(0.5 \mathrm{ml} / 100 \mathrm{~g})$, the hearts were rapidly harvested, rinsed with phosphate buffer and then stepwise dehydrated with 75\%, 85\%, 90\%, 95\% alcohol, anhydrous ethanol, alcoholic benzene, xylene, and wax. The dehydrated tissues were embedded by paraffin, sectioned with a thickness of $4 \mu \mathrm{m}$, dewaxed, and washed with alcohol $(95 \%, 90 \%, 80 \%, 70 \%)$ and distilled water. The sections were stained with hematoxylin and eosin (HE) to observe their morphology.

For the Sirius red staining, the myocardium sections were stained in saturated picric acid Sirius red staining solution, rinsed in anhydrous ethanol, dried, cleared, and finally sealed with neutral gum $[22,23]$.

For Masson's trichrome staining, the sections were stained with Weigert resorcin fuchsin, washed with water, and differentiated with hydrochloric acid alcohol. Then, sections were washed with water, stained blue, stained with Ponceau acid fuchsin, rinsed, and processed with phosphomolybdic acid aqueous solution. Then, sections were restained with aniline blue liquid, processed with glacial acetic acid, and finally sealed with neutral gum after dehydration with alcohol and xylene [24]. Experimental equipment and reagents used for staining pathological section are shown in Additional files 1 and 2 , respectively.

\section{Collagen volume fraction measurement}

Masson staining of the myocardial tissue showed collagen in blue and myocardium in red under microscope $(400 \times)$. Picric acid Sirius red staining showed myocardial fibrin stained in red, and others were yellow. Six nonrepeated fields of view were randomly selected from six different sections of each animal. The myocardial collagen volume fraction (CVF) was measured by Image Pro Plus system. $\mathrm{CVF}=$ collagen area/total area, where the collagen area did not include the perivascular collagen area [24].

\section{Myocardial zymogram}

At the end of NP poisoning, $3 \mathrm{~mL}$ of fasting aortic blood from six rats in each group was randomly taken and serum was collected by centrifugation. Aspartate aminotransferase (AST), serum creatine kinase (CK), serum creatine kinase isozyme (CK-MB), lactate dehydrogenase $(\mathrm{LDH})$ and $\alpha$-hydroxybutyrate dehydrogenase $(\alpha-\mathrm{HBDH})$ were measured by an automatic biochemistry analyzer.

\section{Cardiac Doppler ultrasound}

At the end of NP poisoning, three rats from each group were randomly selected and anesthetized by intraperitoneal injection of $1 \%$ pentobarbital salt solution $(40 \mathrm{mg} /$ $\mathrm{kg})$. A probe of an ultrasound imaging system for small animal models (VEVO 2100) was placed on the left chest of the rats in a supine position. The left ventricular structure was measured, including diastolic thickness of the left ventricular anterior wall (LVAWd), the systolic thickness of the left ventricular anterior wall (LVAWs), left ventricular end diastolic dimensions (LVIDd), left ventricular end systolic dimensions (LVIDs), the diastolic thickness of the left ventricular posterior wall (LVPWd), and the systolic thickness of the left ventricular posterior wall (LVPWs). Each indicator was averaged from three consecutive cardiac cycles [22, 23]. 


\section{Measurement of the expressions of collagen I and collagen III proteins in heart tissue}

At the end of NP poisoning, the hearts were rapidly harvested, lysed by premixed protein lysis solution $(1 \mathrm{~mL}$ RIPA buffer with $10 \mu \mathrm{L}$ phenylmethanesulfonyl fluoride (PMSF)), homogenized, centrifuged to collect the supernatant, and stored after denaturation. Protein concentrations in the hearts of rats were determined by BCA. For electrophoresis, $10 \mu \mathrm{L}$ of sample was loaded at a starting voltage of $80 \mathrm{~V}$. After adequate separation of the marker, the voltage was adjusted at $120 \mathrm{~V}$ to pull bromophenol blue to the bottom of the gel, followed by membrane transfer (continuous current: $300 \mathrm{~mA}, 90 \mathrm{~min}$ ). The membrane was sealed by skimmed milk (5\%) for $2 \mathrm{~h}$, and was incubated with primary antibodies $(\beta$-tubulin, collagen I and collagen III) $(1: 1000)$ for $12 \mathrm{~h}$ at $4{ }^{\circ} \mathrm{C}$ and secondary antibody for $1 \mathrm{~h}$ at $37{ }^{\circ} \mathrm{C}$. The developing solution was dropped on the bands, and further developed by a fusion gel imaging analyzer [25]. The total gray value of an individual band was calculated using image pro Plus (version 6.0). Not cropped WB membrane of collagen type I and collagen type III are shown in Additional file 3.

\section{Statistical analysis}

The data were analyzed by SPSS software (version 18.0), and expressed as mean \pm standard deviation (SD). Oneway ANOVA was used to analyze the difference between groups. The LSD method was used for multiple comparisons of differences between groups when assessing the homogeneity of variance. Dunnett's T3 test was used for multiple comparisons when assessing the heterogeneity of variance. $P<0.01$ was considered to indicate significant differences.

\section{Results}

\section{Accumulated level of NP in the heart}

The NP level in the heart was $10.36 \pm 1.31 \mathrm{ng} / \mathrm{mg}$ in the control group, whereas it was $40.41 \pm 9.79,47.44 \pm 22.24$,

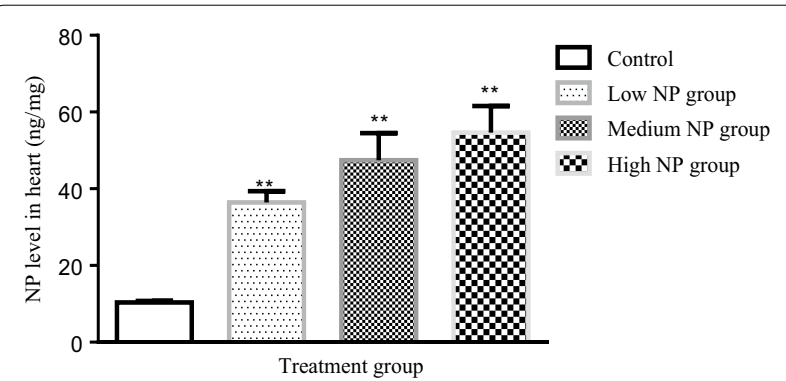

Fig. 2 Comparison of NP levels in the heart between experimental $\operatorname{groups}\left(n=6, \bar{x} \pm s^{* *} P<0.01\right)$ and $54.61 \pm 22.10 \mathrm{ng} / \mathrm{mg}$ in the low, medium, and high NP groups, respectively. These levels were significantly higher than in the control group $(\mathrm{F}=13.704, P<0.01$, Fig. 2).

\section{Morphology observation of myocardial fiber}

Few and regularly distributed pink collagen fibers were detected between normal myocardium. The cardiomyocytes were orderly arranged, dense, and the cardiac muscle fascicles were complete in shape. The low NP group showed a more ordered and looser arrangement, with partial muscle fiber fracture. In the medium and high NP groups, a large amount of fibrous connective tissue was generated around the myocardial tissue and blood vessels. The fibrous connective tissue had a disordered and loose gridding shape. Muscle fibers had fractured (blue arrow), and muscle fibers were loose with a widened gap (red arrow). Extensive inflammatory cell infiltration (black arrow) and deposition of collagen (yellow arrow) in the myocardial interstitium were also found (Fig. 3).

\section{Myocardial fibrosis observation by Masson staining}

In the Masson staining of myocardial tissue, collagen fibers were stained blue and cardiomyocytes were stained are red. Compared with the control group, the low NP group shows a looser arrangement of myocardium with a small amount of blue collagen between myocardium. The medium and high NP group showed a severely disordered arrangement of myocardium, which was loose with a widened myocardial gap. A large amount of blue-stained fibrous connective tissue with a gridded shape enclosed the cardiac muscle fascicle, and a large area blue fibrotic tissue replaced the normal myocardial tissue (Fig. 4). With increasing NP dose, the degree of muscle fiber loosening and disorder became more significant in the NP-treatment groups, and the CVF was higher than that in the control group $(P<0.01$, Fig. 5).

\section{Myocardial fibrosis observation by Sirius red staining}

Type I collagen stained by Sirius red resulted in red staining and others were stained in yellow. Compared with the control group, only few red-stained collagens were observed, and the myocardial gap increased significantly in the low NP group. The medium and high NP groups showed that the arrangement of myocardial fibers was severely disordered, the myocardial interstitial collagen proliferated significantly, a large amount of red-stained fibrous connective tissue in gridded shape enclosed the cardiac muscle fascicles, and a large area of red-stained collagen replaced the normal myocardial tissue. Collagen increased more significantly with 


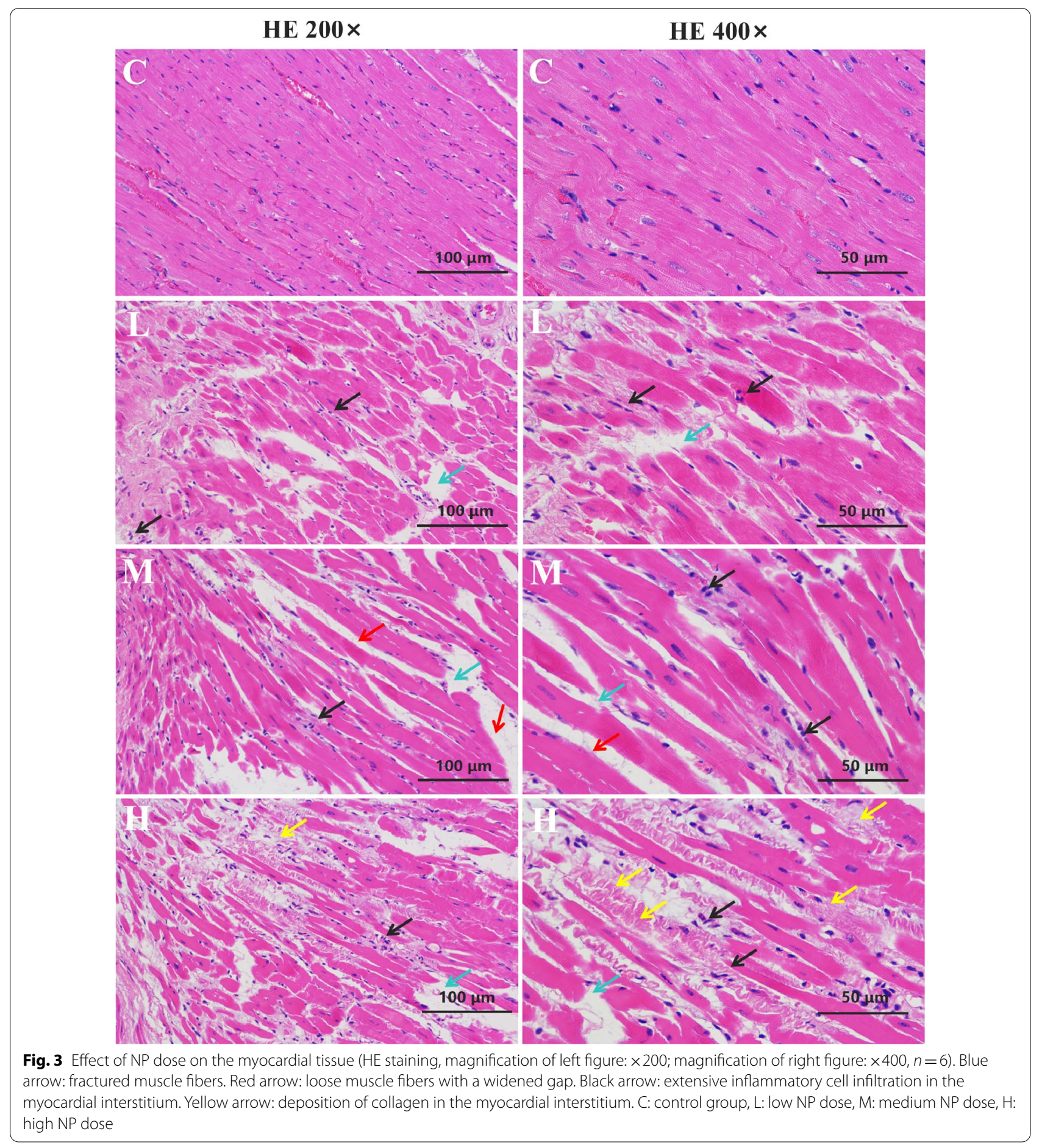




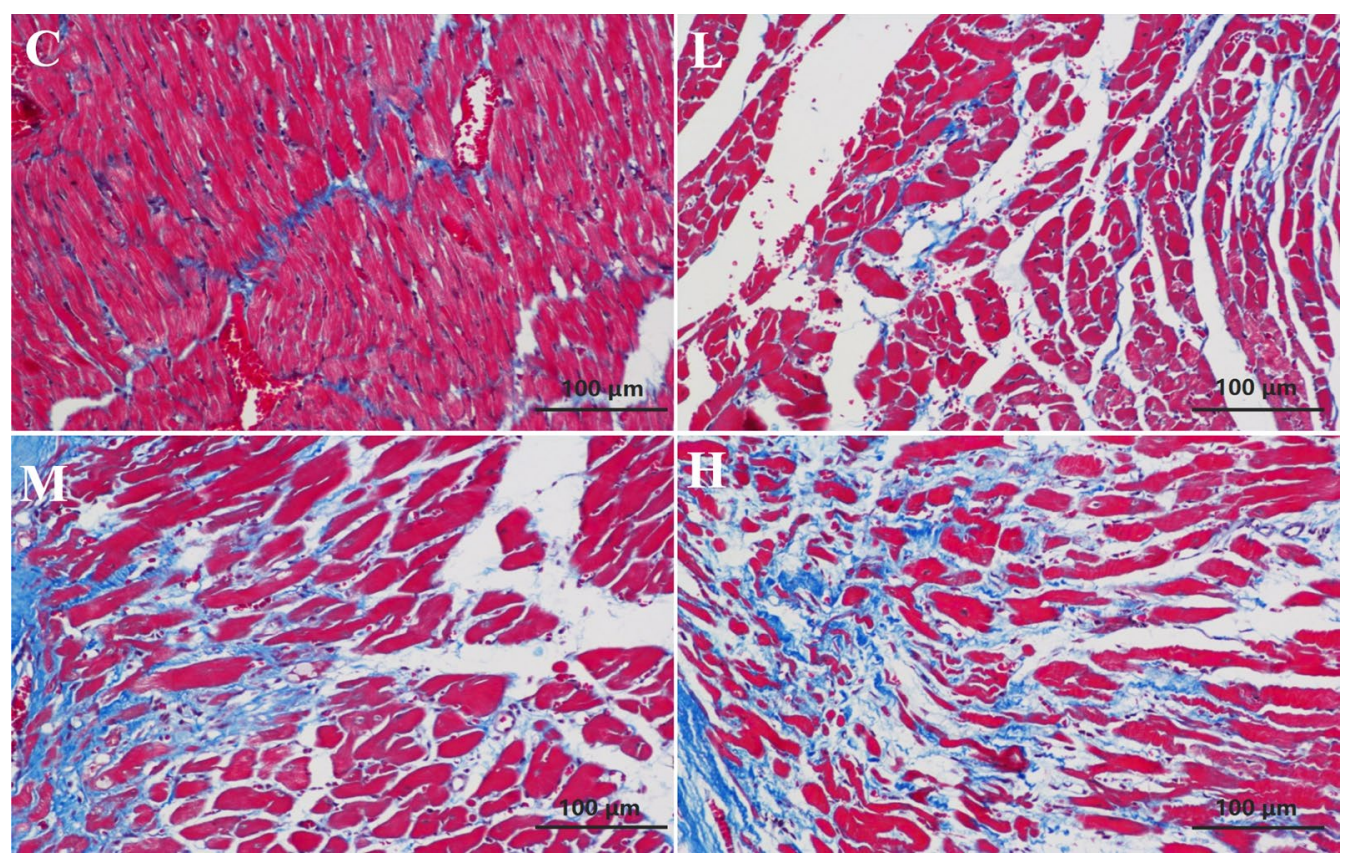

Fig. 4 Myocardial fibrosis induced by different doses of NP (Masson staining, $\times 400, n=6$ ). C: control group, L: low NP dose, M: medium NP dose, H: high NP dose

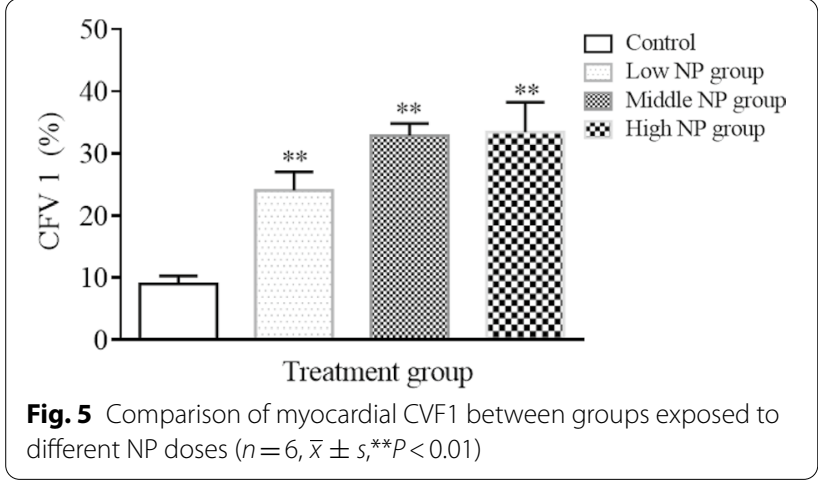

NP dose (Fig. 6). The CVFs in the medium and high NP groups were higher than in the control group $(P<0.01$, Fig. 7).

\section{Effects of NP exposure on serum myocardial enzymes}

Serum AST $(F=43.388, P=0.0001)$, CK $(F=33.456$, $P=0.0001), \quad$ CK-MB $\quad(F=13.929, \quad P=0.0001), \quad$ LDH $(F=78.456, \quad P=0.0001), \quad$ and $\mathrm{a}-\mathrm{HBDH} \quad(F=26.237$, $P=0.0001$ ) significantly increased in the NP groups compared with the control group. These effects increased with NP dose (Fig. 8).
Effects of NP exposure on the left ventricle by cardiac Doppler ultrasound

Compared with the control group, LVAWs were thinner in the medium and high NP groups $(F=16.531, P<0.01$, red arrow). The values of LVPWd, LVPWIs, and LVAWd in the NP groups were slightly lower than those of the control group, and the ventricular wall became thinner. The values of LVIDd in the NP groups increased $(F=9.873$, $P<0.01$, white arrow), the values of LVIDs in the NP groups were slightly higher than in the control group (blue arrow), and the ventricular cavity tended to expand. No significant changes were observed in the left ventricular output and ejection fraction (Figs. 9, 10).

\section{Effects of NP on the expressions of collagen I and collagen III proteins in heart tissue}

As shown in Figs. 11 and 12, compared with the control group, the expression of collagen I and collagen III increased significantly in the medium and high NP groups $(P<0.05)$, increasing gradually with NP dose.

\section{Discussion}

In this study, male SD rats were poisoned by gavage, and the NP content, myocardial fibrosis, cardiac structure, cardiac function, and collagen expression in the 


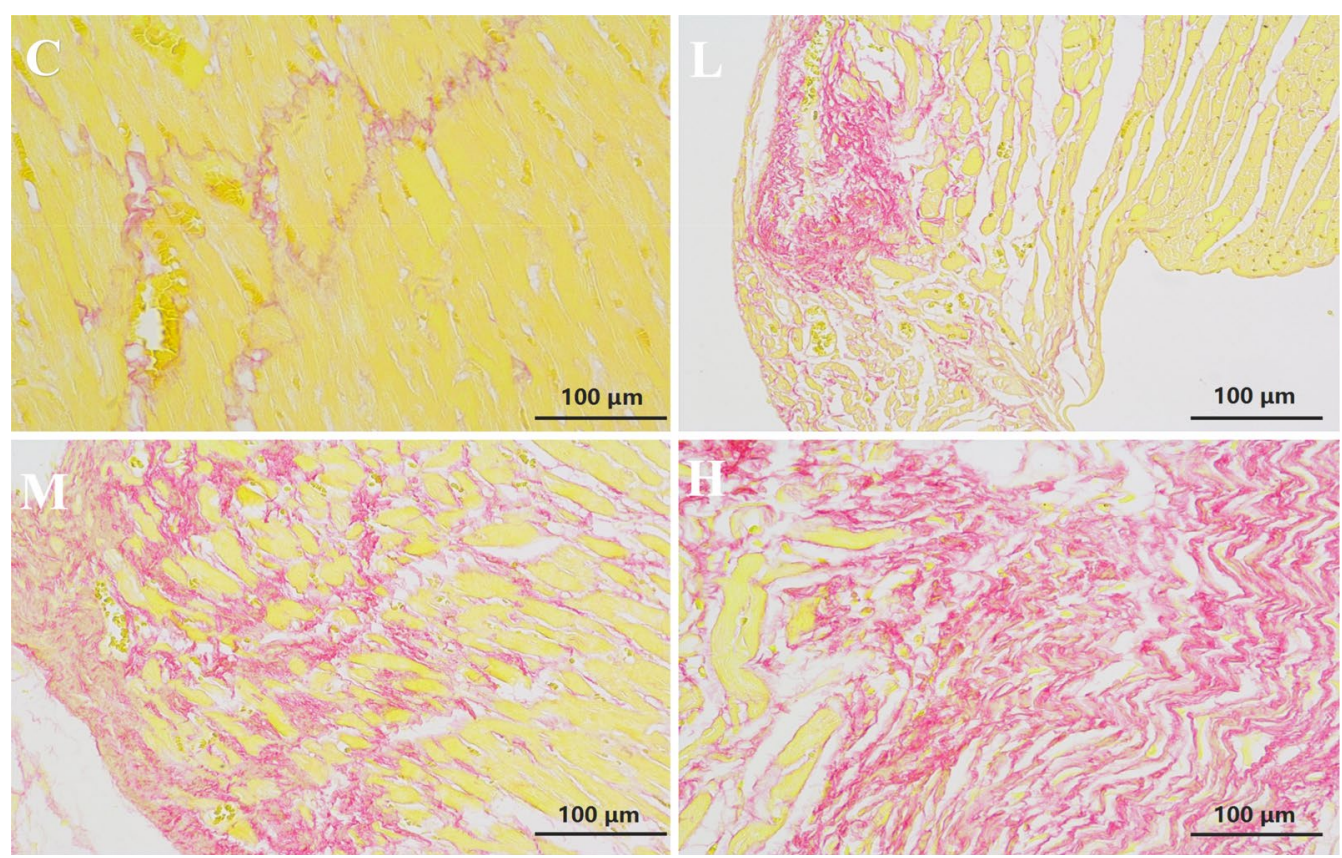

Fig. 6 Myocardial fibrosis induced by different NP doses (Sirius staining, $\times 400, n=6$ ). C: control group, L: low NP dose, M: medium NP dose, H: high NP dose

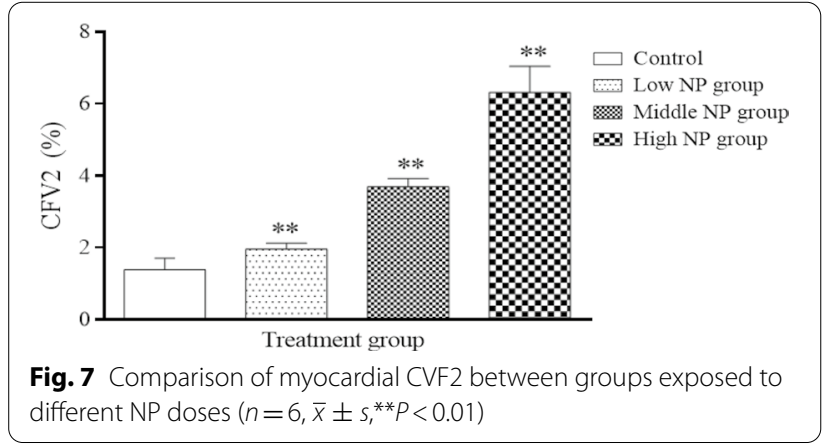

myocardium, as well as changes of cardiac enzymes were examined. For the first time, this study showed that NP is accumulated in the cardiac tissue, leading to myocardial fibrosis, which mainly manifests as increased expression of collagen I and collagen III in the myocardium. This also had adverse effects on the heart, which mainly manifested as alteration of the cardiac structure (i.e., thinning of the ventricular wall and enlargement of the ventricular cavity). These increased levels of cardiac enzymes suggest the damage of the cardiac tissue.

Excessive myocardial fibrosis adversely affects both the myocardial structure and function, which is a common feature of several cardiac diseases, such as hypertension, myocardial infarction, and heart failure [26, 27]. Therefore, the heart was investigated using Doppler ultrasound, which showed a significant thinning of the left ventricular wall at the end systole and a significant increase of the left ventricular end diastolic dimension. At this time, the cardiac output is still at the compensatory stage, and the heart may be undergoing ejection fraction retention heart failure. Myocardial fibrosis is an important factor for the occurrence and development of refractory heart failure. With the progression of fibrosis, the myocardial systolic or diastolic function is further impaired, leading to a declining cardiac pumping function. This in turn leads to the inability to discharge reflux blood to meet the needs of tissue and viscera metabolism, which ultimately causes a series of pathological manifestations [28, 

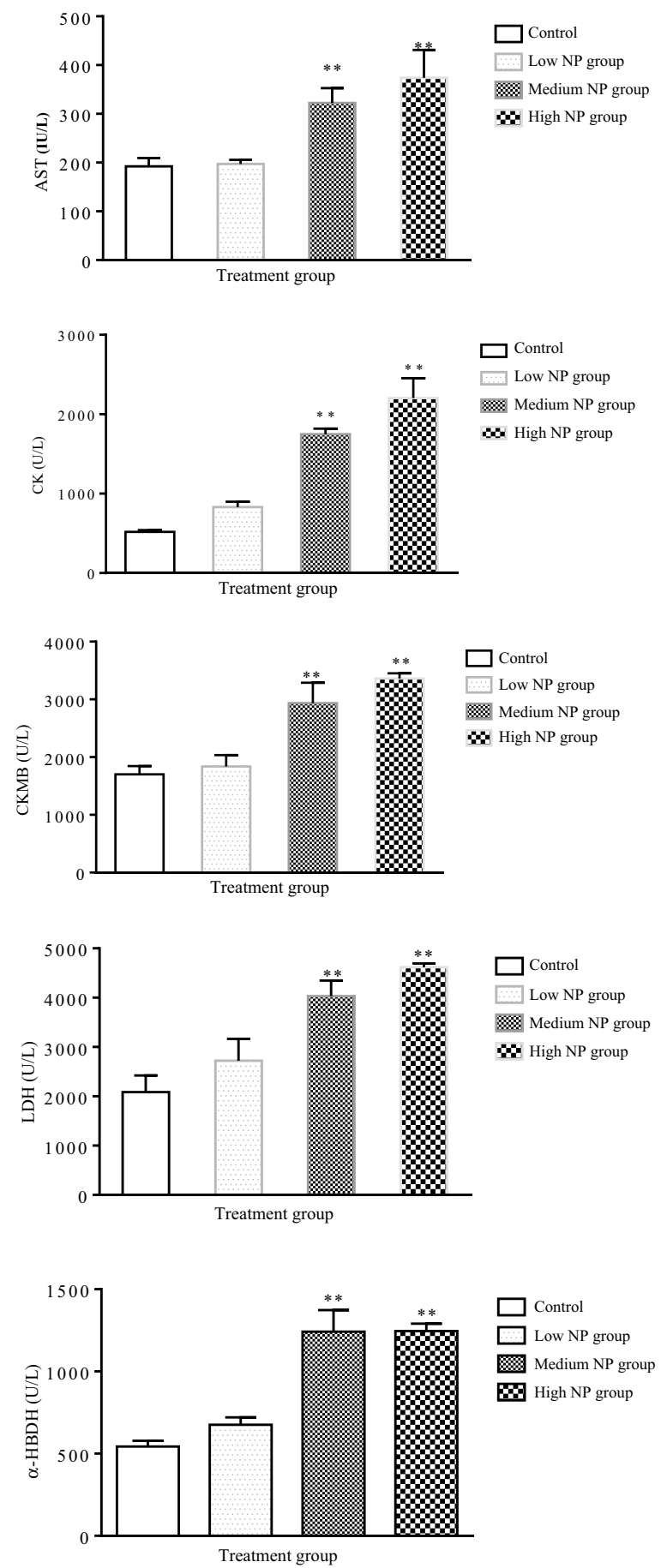

Fig. 8 Comparison of serum myocardial enzyme levels between groups exposed to different NP doses $\left(n=6, \bar{x} \pm s,{ }^{* *} P<0.01\right)$
29]. These interactions are interlinked, and interact with each other in a vicious cycle that ultimately leads to heart damage.

Myocardial fibrosis is capable of altering the myocardial structure, triggering arrhythmias, ischemia, and left ventricular dysfunction. These effects lead to heart failure, ultimately affecting the development and prognosis of cardiac diseases [30-32]. As an important organ of the cardiovascular system, the heart has been reported to be directly poisoned by EEDs, particularly causing myogenic fibrogenic effects. The heart is a target organ of NP, which is highly lipid soluble and accumulates in tissues. Our results show that NP could accumulate in the heart, and more severe myocardial fibrosis will be observed with increasing accumulation dose, indicating that the myocardium may serve as a target organ for the toxic effects of NP.

The detection of myocardial enzymonram can be used as an important factor in the diagnosis of myocardial damage [33, 34]. The results of current study showed that serum levels of LDH, AST, ALT, CK-MB, and $\mathrm{HDBH}$ in NP-treatment groups $(0.4 \mathrm{mg} / \mathrm{kg}$ and $40 \mathrm{mg} / \mathrm{kg}$ ) increased significantly, indicating that the myocardial tissue was damaged. This result is in line with the findings of Wang et al. [35], suggesting that the heart may be the target organ of NP poisoning. The increase of serum myocardial enzymes levels may be related to a certain extent of myocardial damage. Subsequently, HPLC technique was further used to detect NP concentration in myocardial tissue. we found that NP concentration in myocardial tissue was increased in a significant dose-dependent manner, indicating the cumulative toxic effect of NP on heart tissue, and speculating that the heart might a target organ for NP's toxicity, which was consistent with NP's characteristics such as long-term residual toxicity, high bioaccumulation, low metabolic rate in body, etc. In addition, NP was detected in the control group despite its level being lower than that in the NP-treatment group. The NP that detected in the control may originate from water consumption, food intake and/or corn oil, etc., which is associated with the widespread existence of NP in the environment such as domestic water. Our previous study demonstrated that mean NP concentration in terminal tap water was above Standards for the Drinking Water Quality for Phenols of China $(2 \mu \mathrm{g} / \mathrm{L})$. NP in river and tap water in Zunyi city of China had reached the moderate or severe pollution levels [14]. 

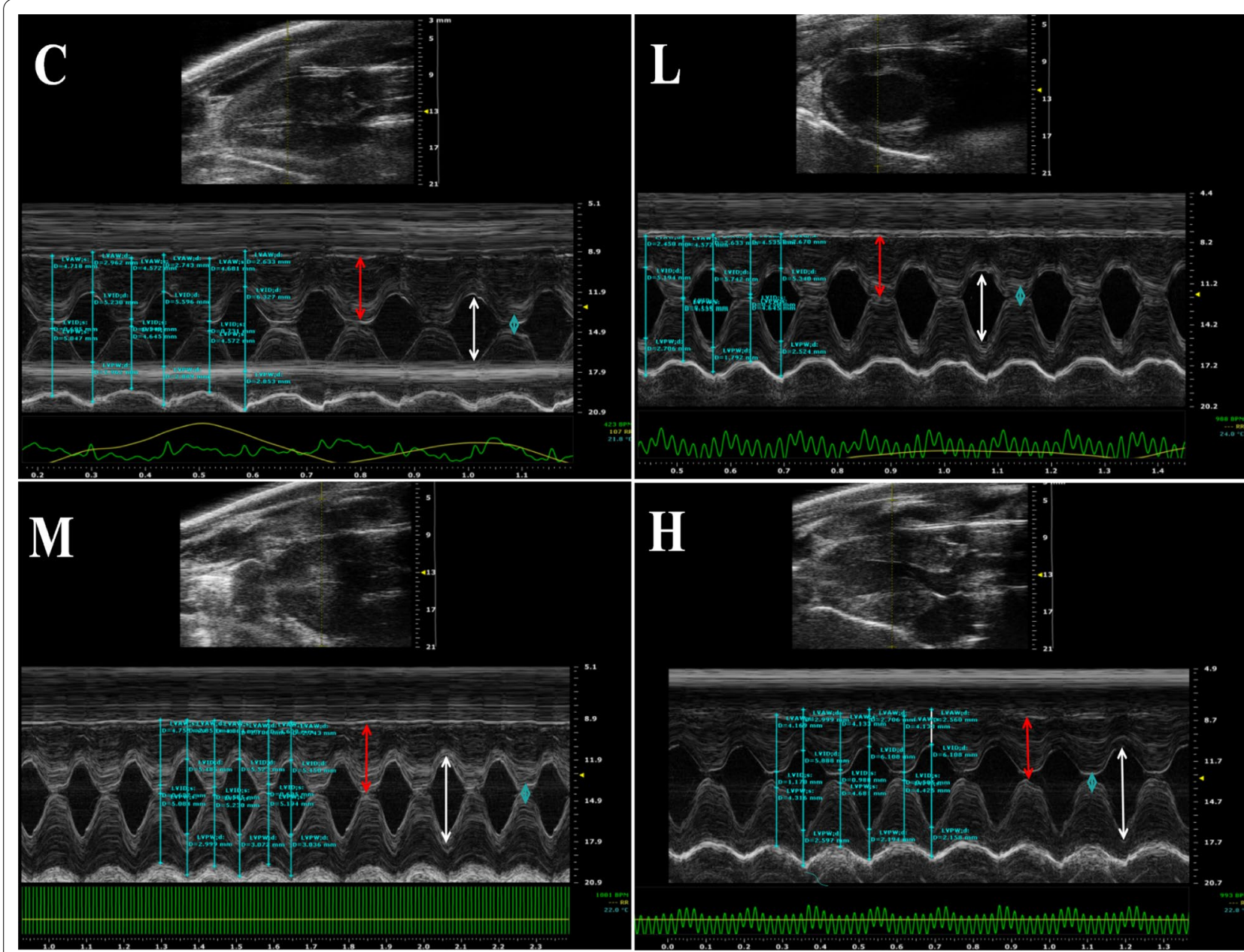

Fig. 9 Effects of NP dose on the left ventricular wall and the lumen of rat hearts. Red arrow: LVAWs. White arrow: LVIDd. Blue arrow: LVIDs. C: control group, L: low NP dose, M: medium NP dose, H: high NP dose

HE staining is an important pathological examination to observe myocardial morphology damage [36]. Therefore, HE staining was conducted to further explore pathological damage in myocardial tissue, which is caused by the accumulation of NP in heart tissue. HE staining of the NP groups showed a large amount of fibrous connective tissue generation in the myocardium and around the blood vessels, and the amount of interstitial collagen in the myocardium increased significantly and thickened. The fibrous connective tissue became disorganized and loosened in a grid-like pattern, and the muscle fibers were fractured. Moreover, a large number of inflammatory cells infiltrated and fibroblasts proliferated in the myocardium, which became more significant with increased NP dose. This indicates that NP can act on myocardial tissue and cause alterations in cardiomyocytes. Based on the pathological alterations in HE staining, we initially confirmed that NP could induce myocardial fibrosis. Subsequently, Sirius red staining and Masson's trichrome staining, which are classical special staining methods used for myocardial fibrosis, were simultaneously used to visualize myocardial fibrosis. Moreover, Sirius red and Masson's trichrome stainings also enabled to measure 

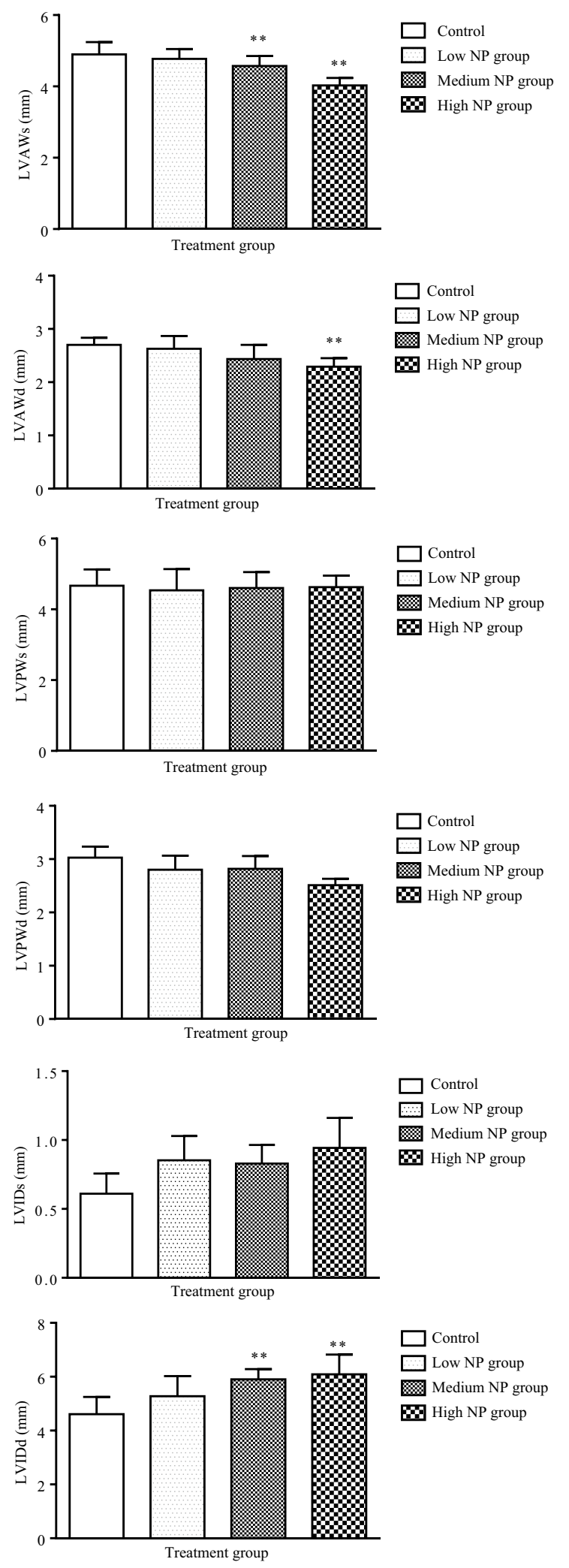

Fig. 10 Comparison of changes of wall thickness and inner diameter of the left ventricle in rats exposed to different NP doses between groups $\left(n=6, \bar{x} \pm s^{* *} P<0.01\right)$ the fraction of the total collagen volume in the myocardium [27, 37-39]. The results showed that a large area of fibrotic tissue replaces normal myocardial tissue in the NP groups. This is consistent with the results of the study on myocardial fibrosis by Song et al. [40], which used Masson staining.

The presence of collagen I and collagen III in the myocardium is a major component of the myocardial collagen network. In order to further confirm the occurrence of myocardial fibrosis, western blot analysis was performed to detect the expression levels of collagen I and collagen III in the myocardial tissues of male rats exposed to NP. The present study showed that the NP-treatment groups had significantly higher levels of myocardial collagen I and collagen III expression, which increased with NP dose. A large number of studies have shown that environmental endocrine disruptors impose harmful effects on the cardiovascular system. For example, BPA can induce myocardial fibrosis by activating the ERK1/2 pathway, thus increasing the proliferation of cardiac fibroblasts and myocardial collagen proliferation [6]. Similarly, the present study showed that the NP-treatment groups had significantly higher levels of myocardial collagen I and collagen III expression, which increased with NP dose. This study demonstrates that NP may contribute to myocardial fibrosis by increasing the collagen I and collagen III expression in the myocardium, which is consistent with the result that $17 \beta$-estradiol induces increased collagen I and collagen III expression in the myocardium [5]. Therefore, we speculated that chronic NP exposure could lead to myocardial fibrosis in male rats.

\section{Conclusion}

Long-term NP exposure can lead to NP accumulation in the cardiac tissue, leading to fibrosis in the rat myocardium, which is characterized by significantly increased myocardial CVF, such as increased expressions of myocardial collagen I and collagen III. These changes increased with increasing NP dose. In addition, the cardiac structure was affected and changes were observed in the thinner ventricular wall and as an enlarged ventricular cavity. NP leads to myocardial fibrosis, elevated cardiac enzymes, and changes in the cardiac structure and function in rats, which are important factors that promote heart failure. This study provides important clues for the prevention and intervention of NP-induced myocardial fibrosis, and it is necessary to study the mechanism and early biomarkers of myocardial fibrosis. 


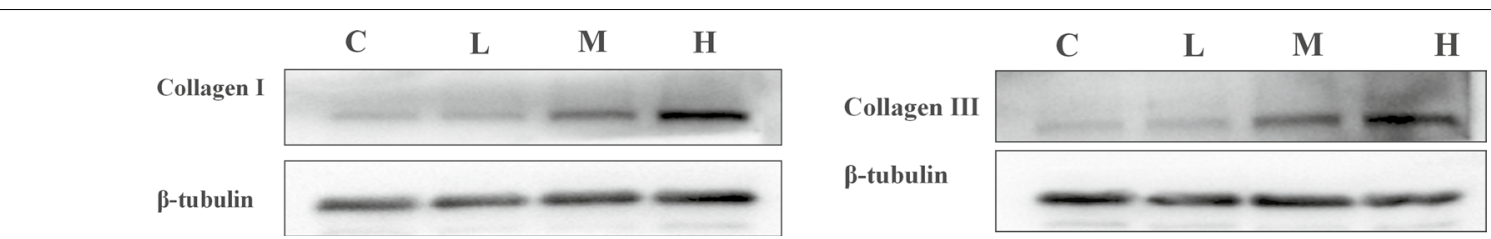

Fig. 11 Expressions of collagen I and collagen III in the myocardial tissues exposed to different NP doses. C: control group, L: low NP dose, M: medium NP dose, $\mathrm{H}$ : high NP dose

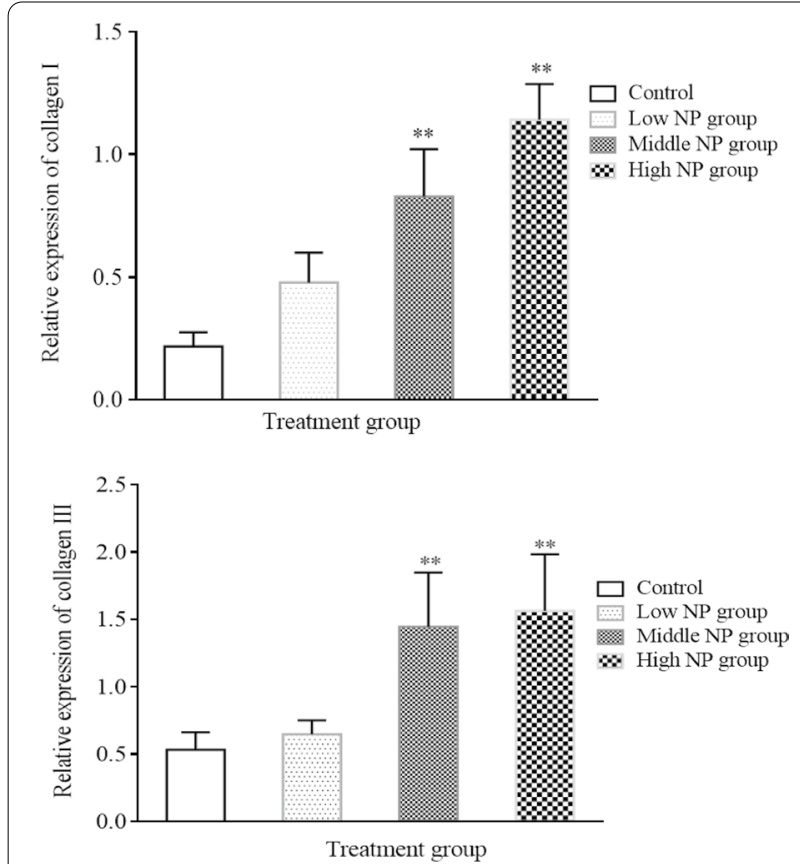

Fig. 12 Comparison of collagen I and collagen III between groups exposed to different NP doses $\left(n=6, \bar{x} \pm s_{1}^{* *} P<0.01\right)$

\section{Abbreviations}

EED: Environmental endocrine disruptors; SD: Sprague-Dawley; HPLC: Highperformance liquid chromatography; AST: Aspartate aminotransferase; CK: Creatine kinase; CK-MB: Creatine kinase isozyme; LDH: Lactate dehydrogenase; a-HBDH: a-Hydroxybutyrate dehydrogenase; CVF: Collagen volume fraction; LVAWs: Left ventricular anterior wall; LVPWd: Left ventricular posterior wall; LVPWs: Left ventricular posterior wall; LVAWd: Left ventricular anterior wall; LVIDs: Left ventricular end systolic dimensions; Collagen I: Collagen Type I; Collagen I: Collagen Type III; H\&E: Hematoxylin-eosin; WB: Western blot; PMSF: Phenylmethanesulfonyl fluoride; PVDF: Polyvinylidene difluoride; RT: Room temperature.

\section{Supplementary Information}

The online version contains supplementary material available at https://doi. org/10.1186/s12302-021-00539-2.

Additional file 1: Table S1. Experimental equipment used for staining pathological section.

Additional file 2: Table S2. Experimental reagents used for staining pathological section.

Additional file 3. Not cropped WB membrane of collagen type I and collagen type III.

\section{Acknowledgements}

Not applicable.

\section{Authors' contributions}

$J X$ and JY designed the study. CL, CN, WL, XY, JZ, RZ, ML, JY and JX analyzed and interpreted the data. CL conducted the laboratory work. JY wrote the manuscript, JX revised the manuscript. All the authors read and approved this paper.

\section{Funding}

This work was supported by the National Natural Science Foundation of China (81760580); the Key Program of Scientific and Technological Fund of Department of Science and Technology of Guizhou Province, China (20191466; 2018-1429); the Scientific and Technological Talent Support Program of the Educational Commission of Guizhou Province of China (KY[2018]054); 15851 Project Talent in Zunyi municipal government, Guizhou Province (2017, 2018(E-262)). Guizhou High-Level Innovative Talent Support Program ([2020]6014). Undergraduate innovation and entrepreneurship training program of Zunyi Medical University (ZYDC2018041).

\section{Availability of data and materials}

The datasets used and/or analyzed during the current study available from the corresponding author on reasonable request.

\section{Declarations}

Ethics approval and consent to participate

The Ethics Committee of the Zunyi Medical University approved the study (No: 2015-2-011). All methods were performed in accordance with guidelines and regulations of the Zunyi Medical University.

\section{Consent for publication}

All the authors read and approved this paper.

\section{Competing interests}

The authors declare that they have no competing interests.

\section{Author details}

${ }^{1}$ School of Public Health, Zunyi Medical University, Zunyi, Guizhou 563000 People's Republic of China. ${ }^{2}$ Department of Cardiology, Affiliated Hospital of Zunyi Medical University, Guizhou, People's Republic of China.

Received: 5 January 2021 Accepted: 31 July 2021

Published online: 18 August 2021

\section{References}

1. Działo E, Tkacz K, Błyszczuk P (2018) Crosstalk between the TGF- $\beta$ and WNT signalling pathways during cardiac fibrogenesis. Acta Biochim Pol 65(3):341-349

2. Delaunay M, Halima O et al (2020) The role of cyclic AMP signaling in cardiac fibrosis. Cells 9(1):1-22

3. González A, López B, Ravassa S et al (2019) The complex dynamics of myocardial interstitial fibrosis in heart failure. Focus Collagen Cross Link. 1866(9):1421-1432 
4. González A, Schelbert EB, Díez J et al (2018) Myocardial interstitial fibrosis in heart failure: biological and translational perspectives. J Am College Cardiol. 71(15):1696-1706

5. Dworatzek E, Mahmoodzadeh S, Schriever C, Kusumoto K, Kramer L, Santos G, Fliegner D, Leung YK, Ho SM, Zimmermann WH, Lutz S, RegitzZagrosek V (2019) Sex-specific regulation of collagen I and III expression by 17ß-Estradiol in cardiac fibroblasts: role of estrogen receptors. Cardiovasc Res 115(2):315-327

6. Hu Y, Zhang L, Wu X, Hou L, Li Z, Ju J, Li Q, Qin W, Li J, Zhang Q, ZhouT, Zhang L, Xu C, Fang Z, Zhang Y, Bisphenol A (2016) An environmental estrogen-like toxic chemical, induces cardiac fibrosis by activating the ERK $1 / 2$ pathway. Toxicol Lett 27(250-251):1-9

7. Medina-Buelvas DM, Estrada-Muñiz E, Rodríguez-Sosa M et al (2019) Increased heart fibrosis and acute infection in a murine Chagas disease model associated with organophosphorus pesticide metabolite exposure. Sci Rep 9(1):17539

8. Feriani A, Tir M, Gómez-Caravaca AM, Contreras MDM et al (2020) HPL C-DADESI-QTOF-MS/MS profiling of Zygophyllum album roots extract and assessment of its cardioprotective effect against deltamethrin-induced myocardial injuries in rat, by suppression of oxidative stress-related inflammation and apoptosis via NF-kB signaling pathway. J Ethnopharmacol. 247:112266

9. Ozmen O (2013) Cardiotoxicity and apoptotic activity in subacute endosulfan toxicity and the protective effect of vitamin C in rabbits: a pathological study. J Environ Pathol Toxicol Oncol 32(1):53-58

10. James $\mathrm{LC}$, Paolo B, Warren FG, et al. Comments on the opinions published by Bergman et al. (2015) on Critical Comments on the WHO-UNEP State of the Science of Endocrine Disrupting Chemicals (Lamb et al., 2014). 2015, 73(3):754-7.

11. Alberto M (2016) Endocrine disrupters and the safety of food chains. Horm Res Paediatr 86(4):279-288

12. Sheikh IA, Tayubi IA, Ahmad E et al (2017) Computational insights into the molecular interactions of environmental xenoestrogens 4-tert-octylphenol, 4-nonylphenol, bisphenol A (BPA), and BPA metabolite, 4-methyl-2, 4-bis (4-hydroxyphenyl) pent-1-ene (MBP) with human sex hormone-binding globulin. Ecotoxicol Environ Saf 135:284-291

13. Melzer D, Osborne NJ, Henley WE et al (2012) Urinary bisphenol A concentration and risk of future coronary artery disease in apparently healthy men and women. Circulation. 5(12):1482-1490

14. Jie Y, Jie Z, Xu J et al (2017) Pollution by Nonylphenol in river, tap water, and aquatic in an acid rain-plaqued city in southwest China. Int J Environ Health Res. 27(3):179-190

15. Lee J, Choi K, Park J et al (2018) Bisphenol A distribution in serum, urine, placenta, breast milk, and umbilical cord serum in a birth panel of motherneonate pairs. Sci Total Environ 626:1494-1501

16. Azzouz A, Rascón AJ, Ballesteros E (2016) Simultaneous determination of parabens, alkylphenols, phenylphenols, bisphenol A and triclosan in human urine, blood and breast milk by continuous solid-phase extraction and gas chromatography-mass spectrometry. J Pharm Biomed Anal 119:16-26

17. Gao Q, Liu S, Guo F et al (2015) Nonylphenol affects myocardial contractility and L-type $\mathrm{Ca}(2+)$ channel currents in a non-monotonic manner via $\mathrm{G}$ protein-coupled receptor 30. Toxicology 334:122-129

18. Shen H, Hu Y, Liu X, Jiang Z, Ye H. Role of Cardiovascular Color Doppler Imaging Information Technology Under Artificial Intelligence Neural Network in the Diagnosis of Septic Shock Patients (published online ahead of print, 2020 Jun 22). Neurosci Lett. 2020;135199.

19. Sudharshana Murthy KA, Ashoka HG, Aparna AN (2016) Evaluation and comparison of biomarkers in heart failure. Indian Heart J. 68(Suppl 1):S22-S28

20. Wang Z, Ding J, Luo X et al (2016) Effect of allopurinol on myocardial energy metabolism in chronic heart failure rats after myocardial infarct. Int Heart $J$ 57(6):753-759

21. Guo C, Zhang J, Zhang P et al (2019) Ginkgolide B ameliorates myocardial ischemia reperfusion injury in rats via inhibiting endoplasmic reticulum stress. Drug Des Devel Ther 13:767-e774
22. Ribeiro S, Pereira AR, Pinto AT, Rocha F, Ministro A, Fiuza M, Pinto F, Santos SC (2019) Echocardiographic assessment of cardiac anatomy and function in adult rats. J Vis Exp. 154:60404

23. Boluyt MO, Converso K, Hwang HS, Mikkor A, Russell MW (2004) Echocardiographic assessment of age-associated changes in systolic and diastolic function of the female F344 rat heart. J Appl Physiol (1985). 96(2):822-8

24. Rittié $L$ (2017) Method for picrosirius red-polarization detection of collagen fibers in tissue sections. Methods Mol Biol 1627:395-407

25. Lee MH, Park HW, Kim MH (2006) Efficient cellular uptake of recombinant murine Hoxc8 homeoprotein in COS-7 cells. Life Sci 79(25):2345-2348

26. Wang Y, Hu H, Zhao M, Zhao J, Yin D, Sun X, Liu S, Gao Q, Yu L, Hao L (2013) Nonylphenol disrupts the cardio-protective effects of $17 \beta$-estradiol on ischemia/reperfusion injury in isolated hearts of guinea pig. J Toxicol Sci 38(5):731-740

27. Fan D, Takawale A, Lee J, Kassiri Z (2012) Cardiac fibroblasts, fibrosis and extracellular matrix remodeling in heart disease. Fibrogenesis Tissue Repair 5(1):15

28. Zhao Y, Ding C-H (2018) In vitro effects of nerve growth factor on cardiac fibroblasts proliferation. Cell Cycle Migration Myofibroblast Transform 131(7):813-817

29. Ravassa S, López B, Querejeta R et al (2017) Phenotyping of myocardial fibrosis in hypertensive patients with heart failure. Influence Clin Outcome J Hypertens 35(4):853-861

30. Jiang Y, Wang J, Wang Y, Ke X, Zhang C, Yang R (2018) Self-emulsifying drug delivery system improves preventive effect of curcuminoids on chronic heart failure in rats. Saudi Pharm J 26(4):528-534

31. González A, Schelbert EB, Díez J, Butler J (2018) Myocardial interstitial fibrosis in heart failure: biological and translational perspectives. J Am Coll Cardiol 71(15):1696-1706

32. López B, González A, Ravassa S, Beaumont J, Moreno MU, San José G, Querejeta R, Díez J (2015) Circulating biomarkers of myocardial fibrosis: the need for a reappraisal. J Am Coll Cardiol 65(22):2449-2456

33. Marshall L, Vivien C, Girardot F et al (2017) Persistent fibrosis, hypertrophy and sarcomere disorganisation after endoscopy-guided heart resection in adult Xenopus. PLoS One. 12(3):e0173418

34. Zhang J, Zhou Y, Sun Y et al (2019) Beneficial effects of Oridonin on myocardial ischemia/reperfusion injury: insight gained by metabolomic approaches. Eur J Pharmacol. 861:172587

35. Wang Y, Hu H, Zhao M et al (2013) Nonylphenol disrupts the cardio-protective effects of $17 \beta$-estradiol on ischemia/reperfusion injury in isolated hearts of guinea pig. JToxicol Sci 38(5):731-740

36. Jiang Y, Wang J, Wang Y et al (2018) Self-emulsifying drug delivery system improves preventive effect of curcuminoids on chronic heart failure in rats. Saudi Pharm J 26(4):528-534

37. Zhang LX, Zhang SH, Wang CQ et al (2019) Role and mechanism of microRNA-548c-3p/c-Myb in myocardial infarction fibrosis in rats. Eur Rev Med Pharmacol Sci 23(11):4908-4916

38. Gao S, Li L, Li L et al (2019) Effects of the combination of tanshinone IIA and puerarin on cardiac function and inflammatory response in myocardial ischemia mice. J Mol Cell Cardiol 137:59-70

39. Song LL, Zhang $Y$, Zhang $X R$ et al (2019) Theacrine attenuates myocardial fibrosis after myocardial infarction via the SIRT3/ $\beta$-catenin/PPARY pathway in estrogen-deficient mice. Eur Rev Med Pharmacol Sci 23(12):5477-5486

40. Song LL, Zhang Y, Zhang XR, Song YN, Dai HZ (2019) Theacrine attenuates myocardial fibrosis after myocardial infarction via the SIRT3/ $\beta$-catenin/ PPARy pathway in estrogen-deficient mice. Eur Rev Med Pharmacol Sci 23(12):5477-5486

\section{Publisher's Note}

Springer Nature remains neutral with regard to jurisdictional claims in published maps and institutional affiliations. 